\title{
Temporal and Spatial Variability in Sediment Texture on Northern, Paraglacial Beaches: New \\ Hampshire
}

\section{Imini}
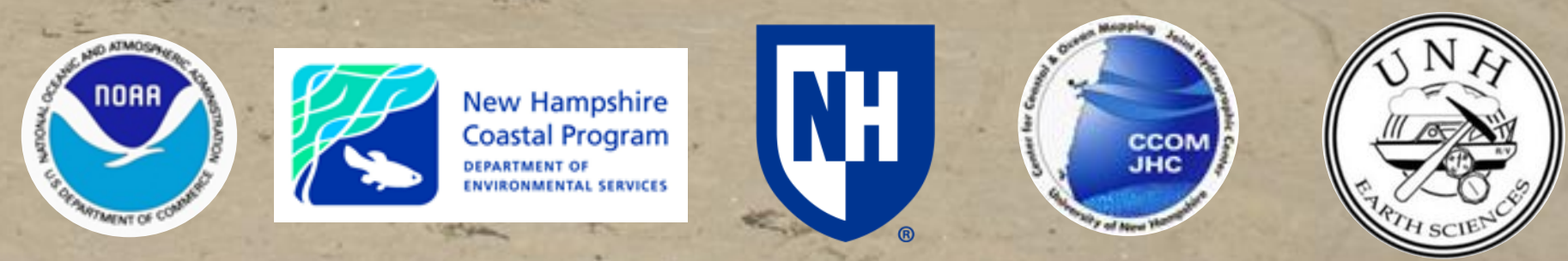


\section{Project Goals}

- Determine the textural characteristics of the New Hampshire beaches

- Determine temporal variability of sediments

- Assess the source of sediment 


\section{Motivation}

- Sea-Level Rise

- Coastal Resiliency

- Beach Nourishment

- First Detailed

Sediment Study on

New Hampshire Coast

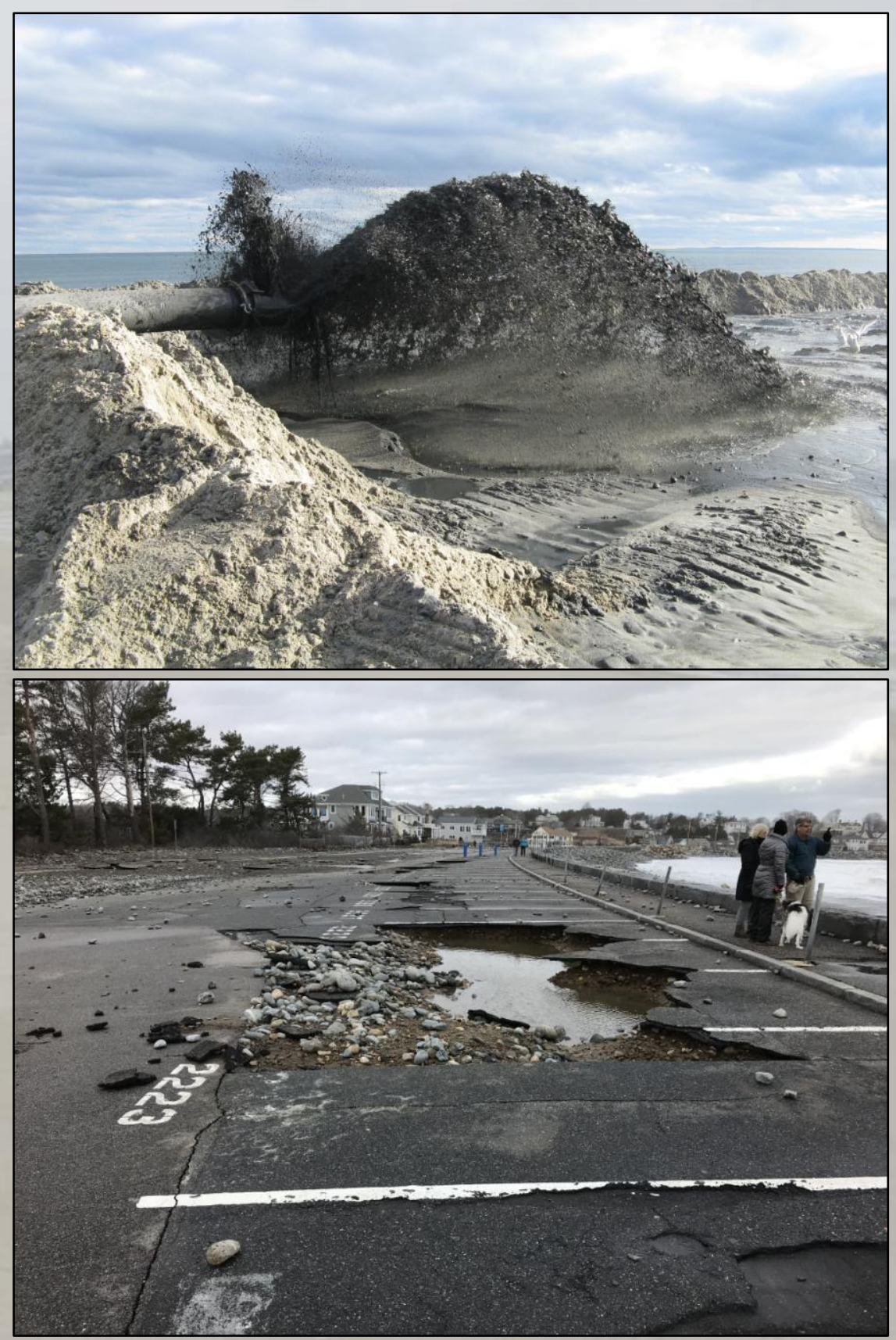




\section{Study Area}

- Seven main NH beaches

- Includes small pocket beaches, attached barriers, and a barrier island

- Mostly separated by headlands

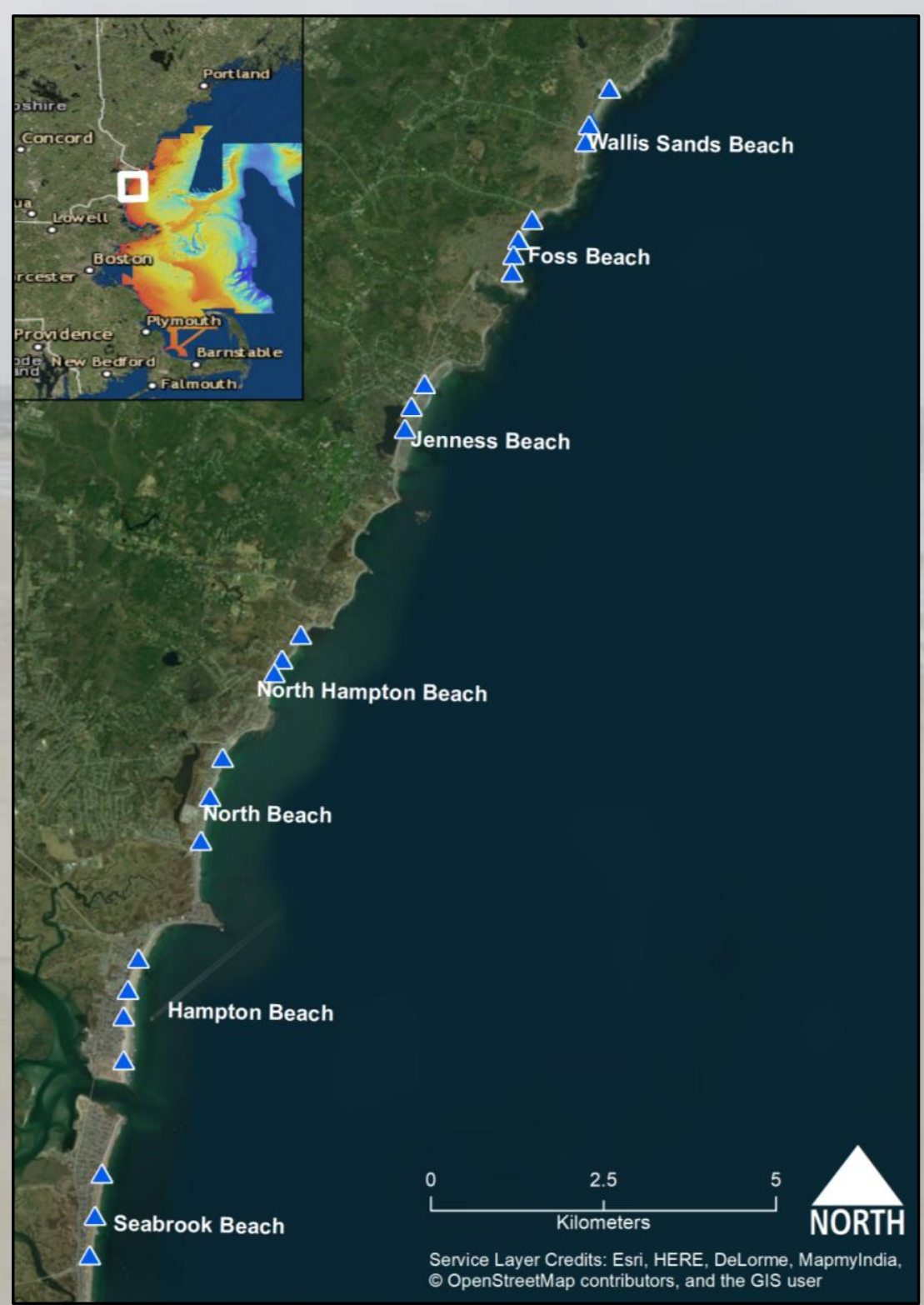




\section{Methods}

- 24 transects on 7 main beaches

- Sampled after extended erosional and accretional periods

- At least 4 samples taken per transect

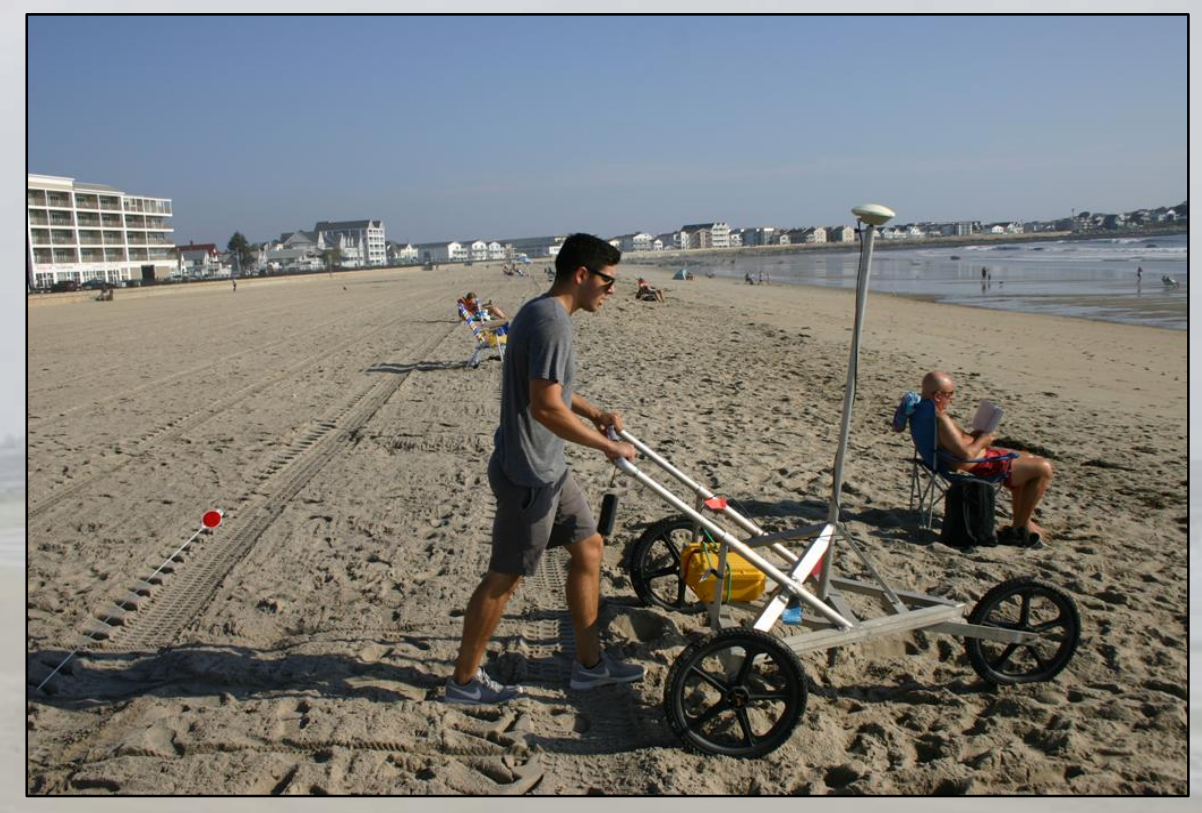

- Over 240 samples in total

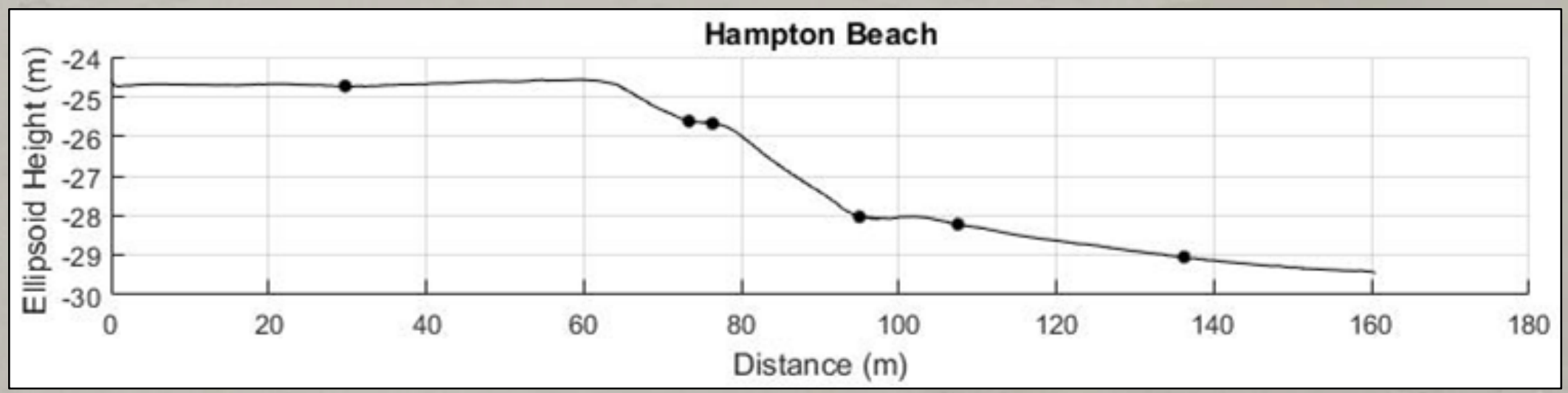




\section{Sampling}
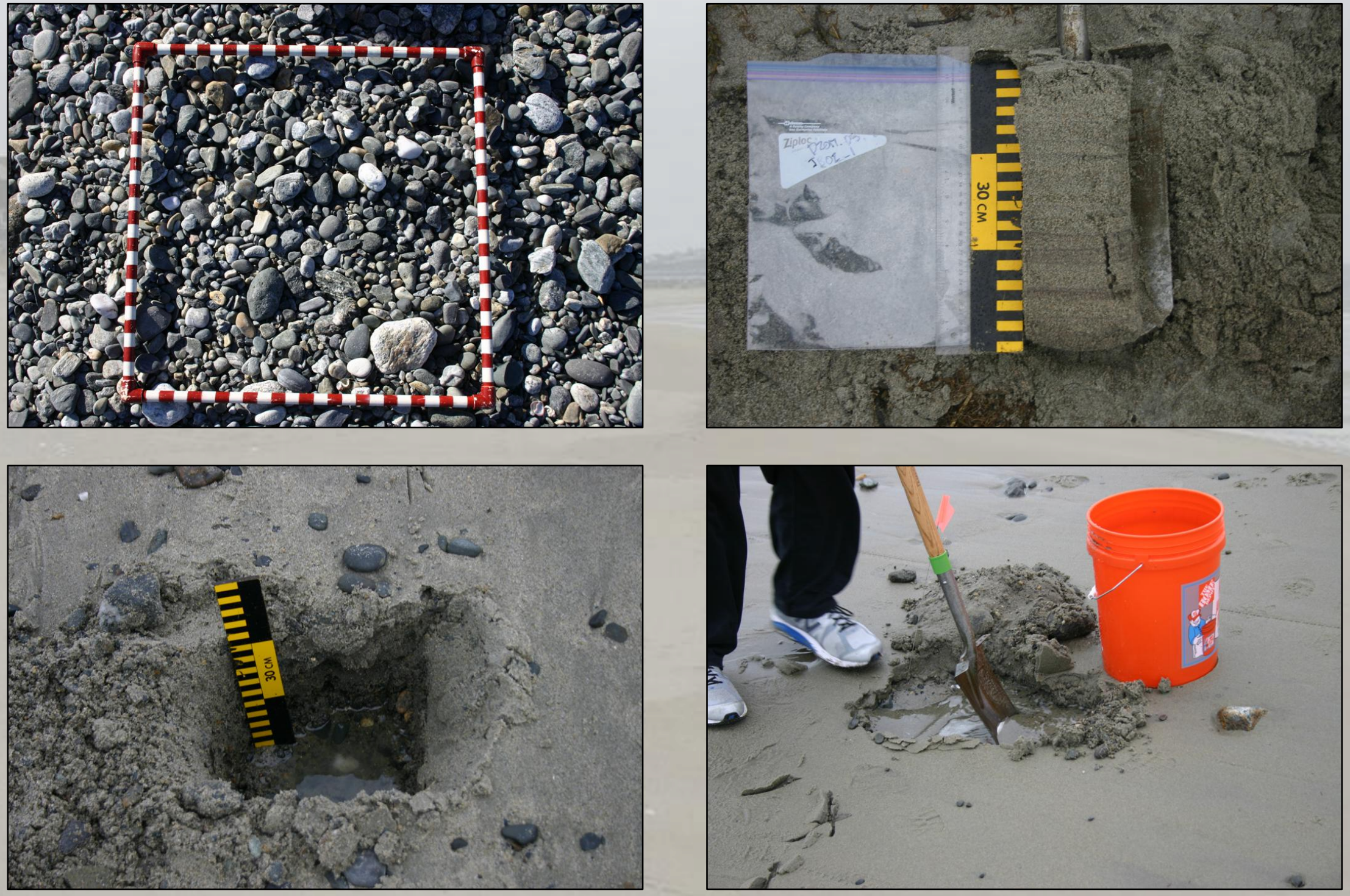


\section{Sediment Sources}

- Headlands Cut Off Longshore Transport - Minimal River Input - Glacially Sourced Sediment

- Headlands - Offshore

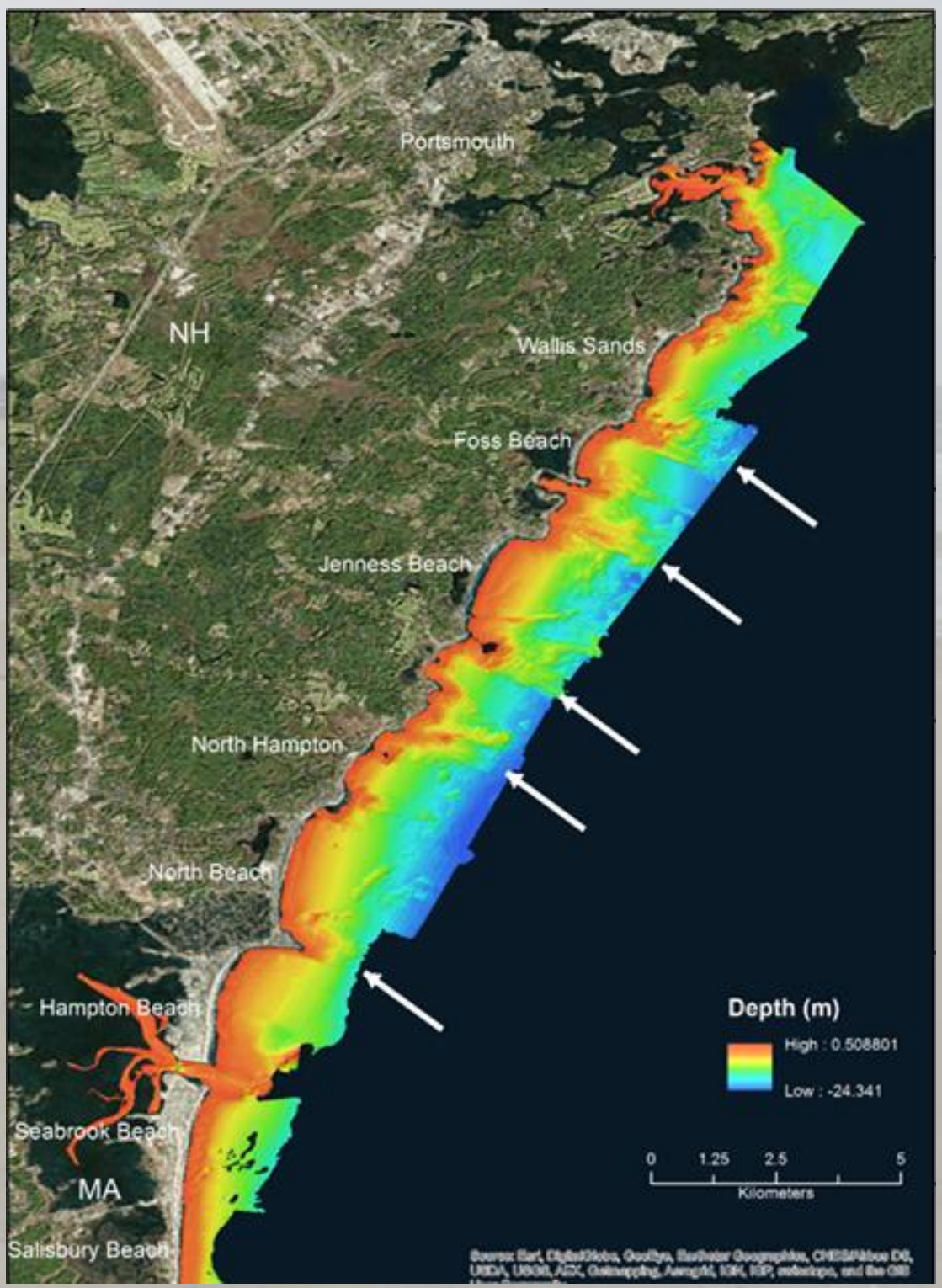




\section{Surficial Geology}
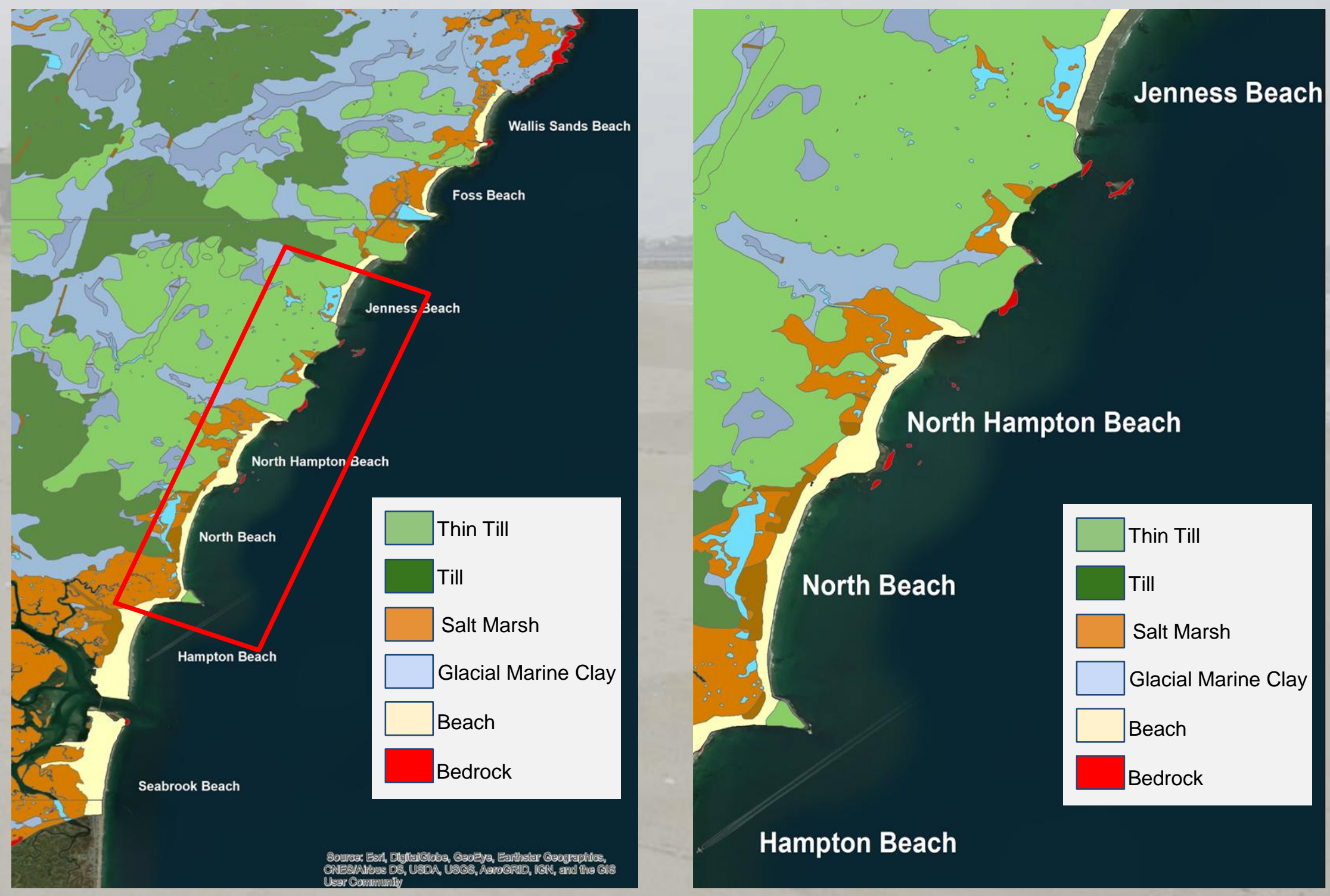


\section{Offshore Sediment}

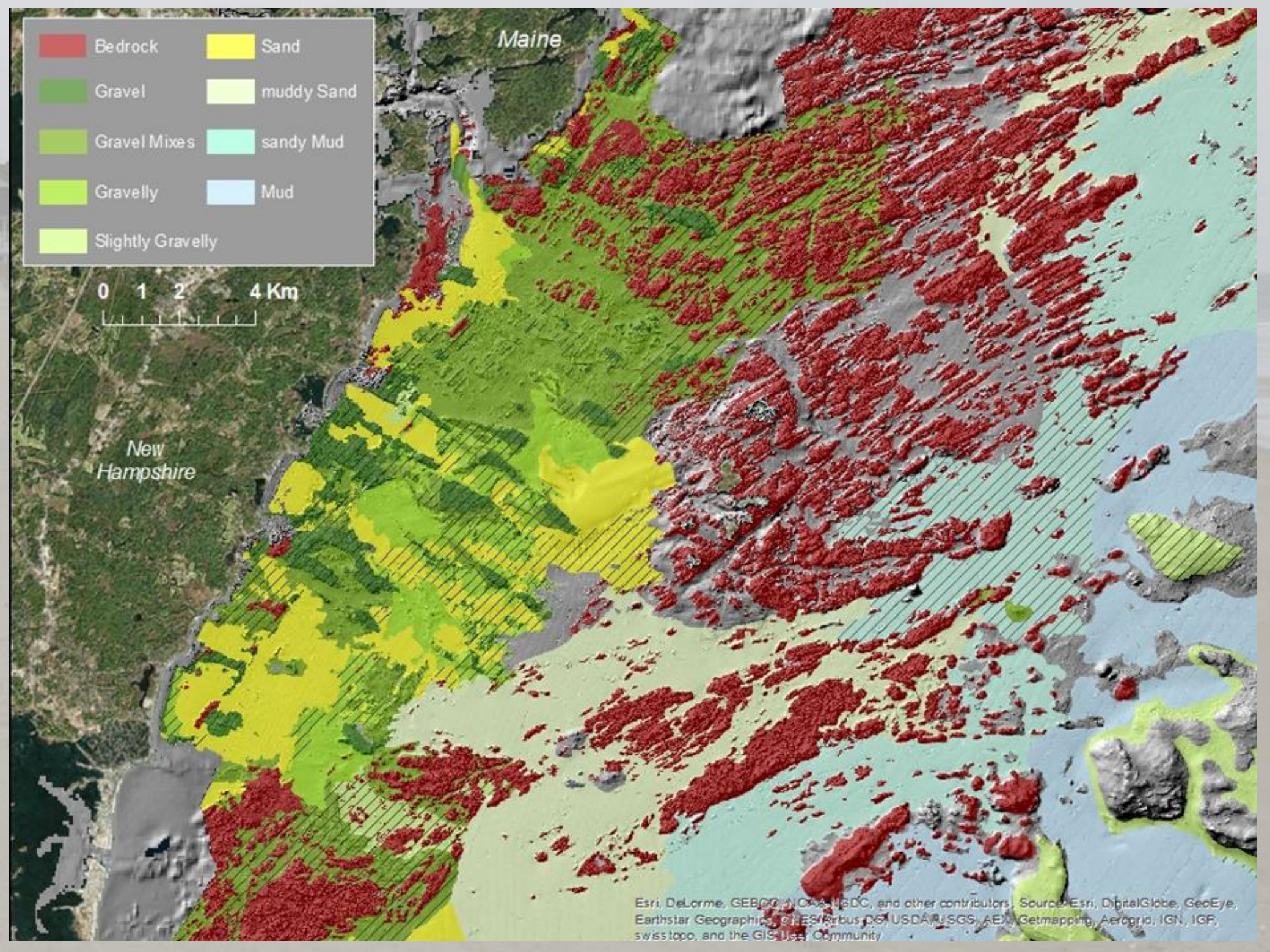




\section{Characteristics of Beach Sediments}

- Northern Beaches

- Bimodal; Sand-Cobble

- Highly Variable

- Southern Beaches

- Unimodal

- Medium to Coarse Sand

North Hampton (August 8, 2016):

Accretional Conditions

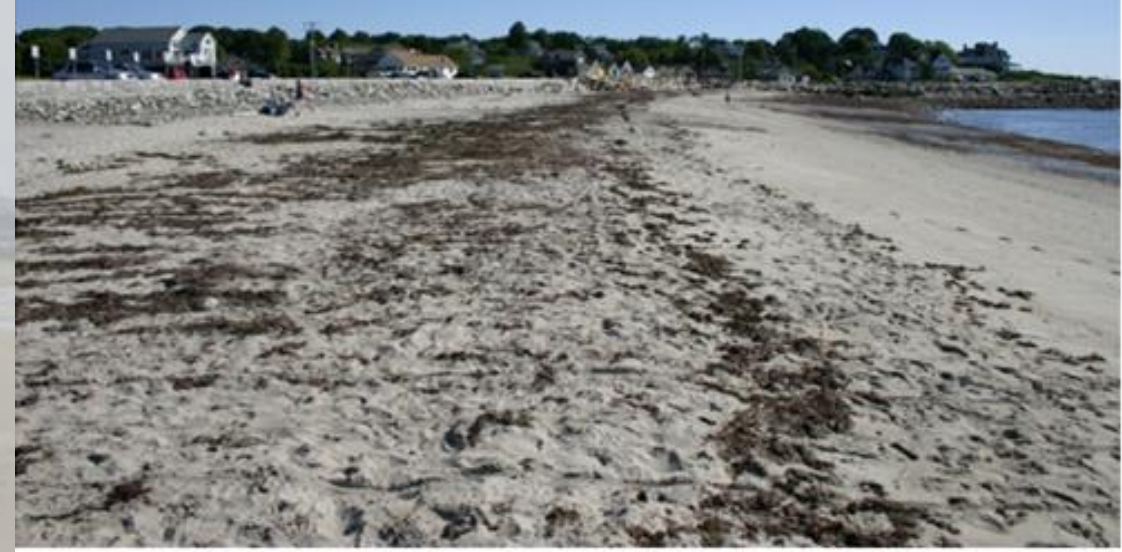

North Hampton (September 9, 2016):

Erosional Conditions (after Hermine)

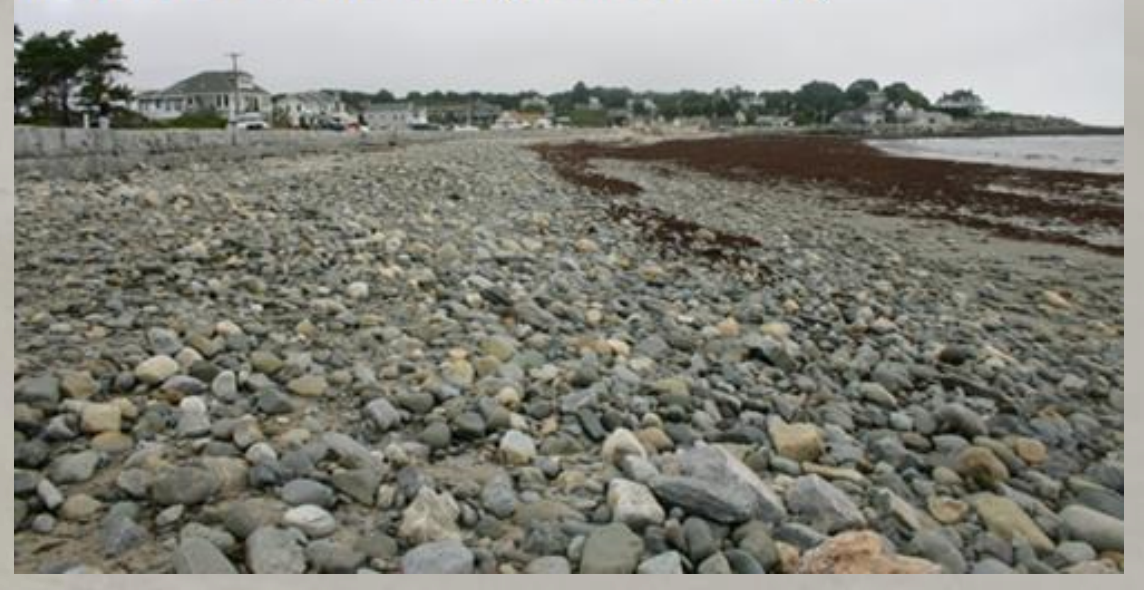




\section{Northern Beaches: Wallis Sands}

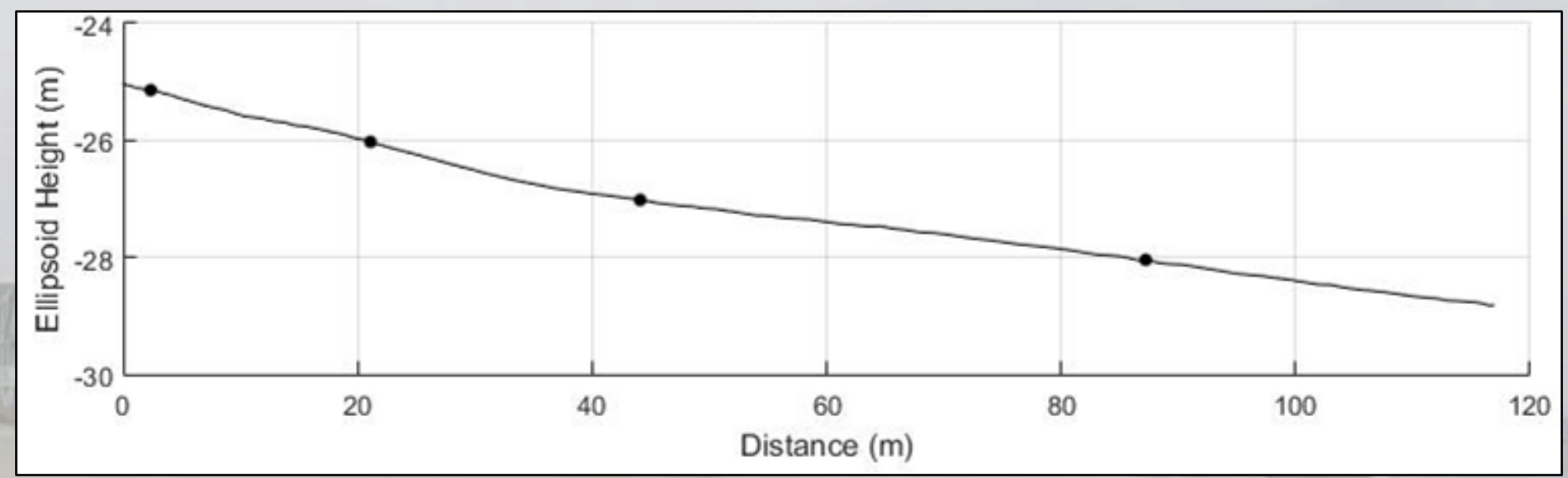

Station 2- Representative Profile
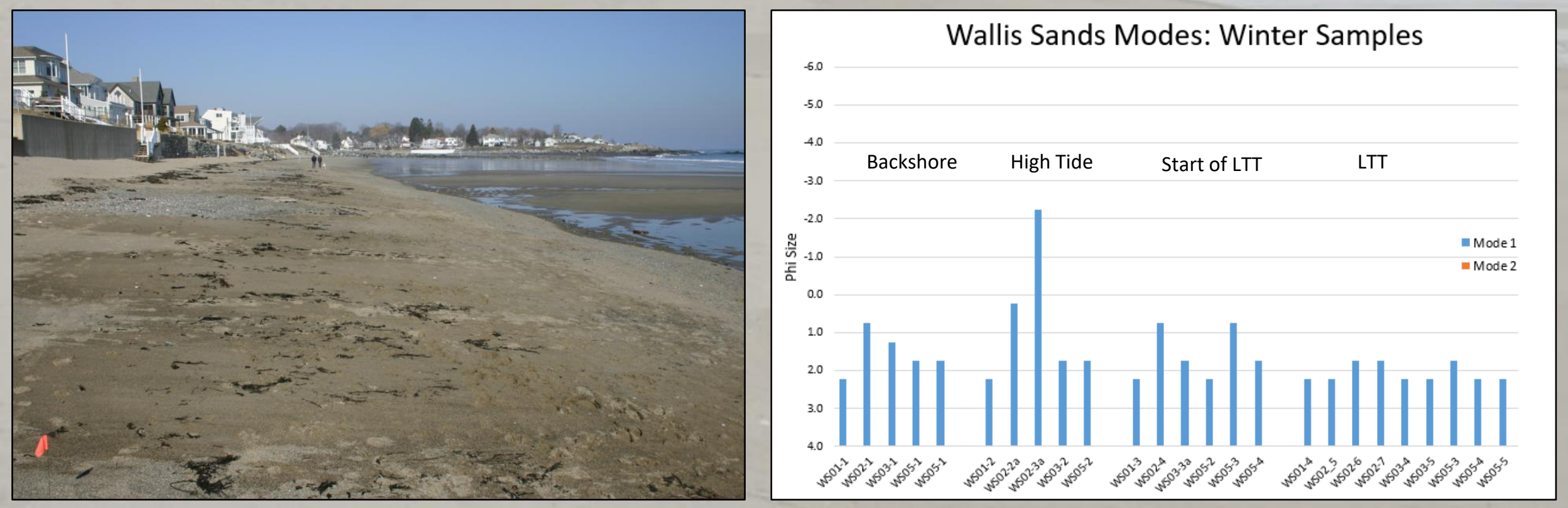


\section{Northern Beaches: North Hampton Beach}
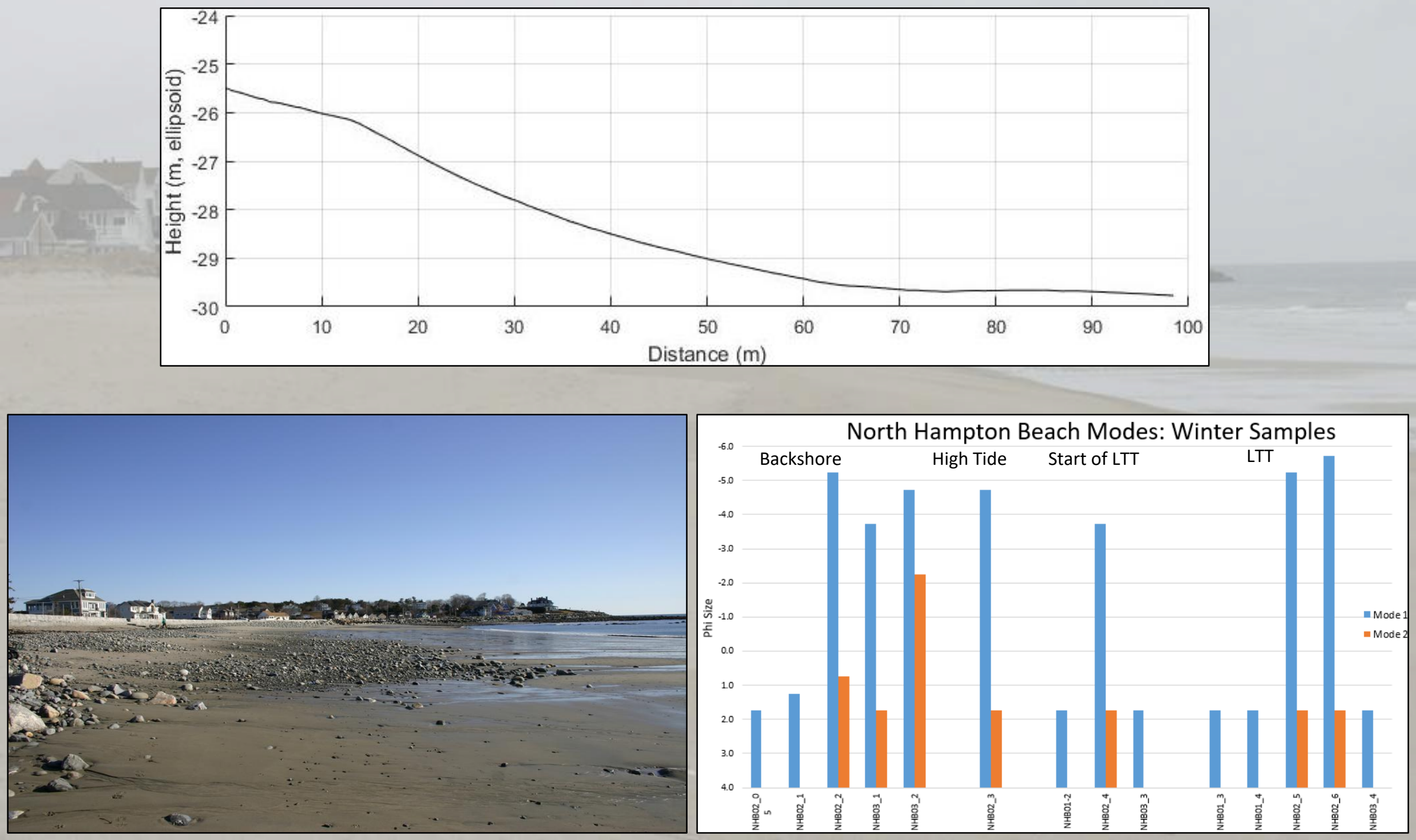


\section{Southern Beaches: Hampton Beach}
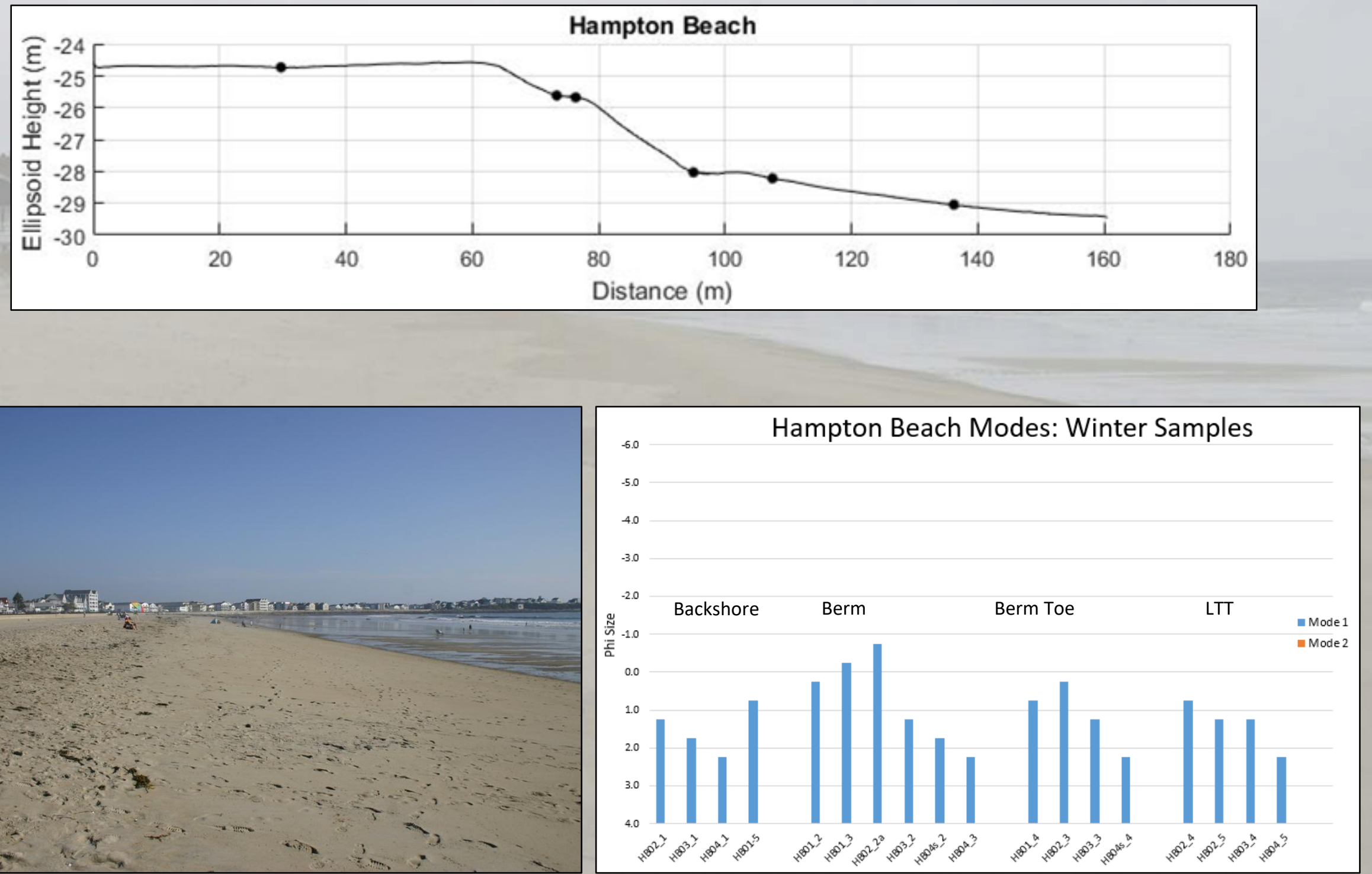


\section{Single Profile Variation}

- There can be drastic changes along a single profile
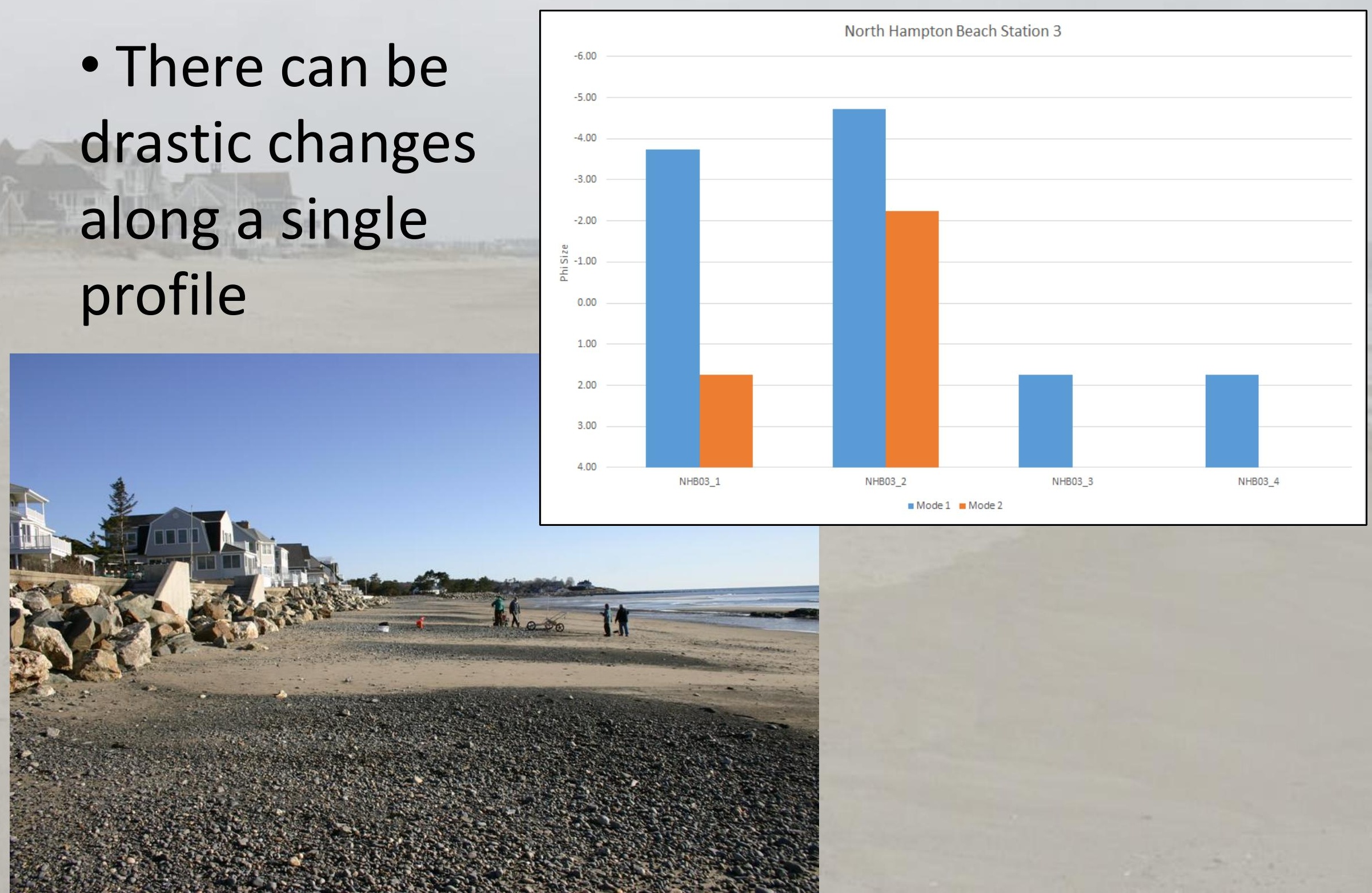

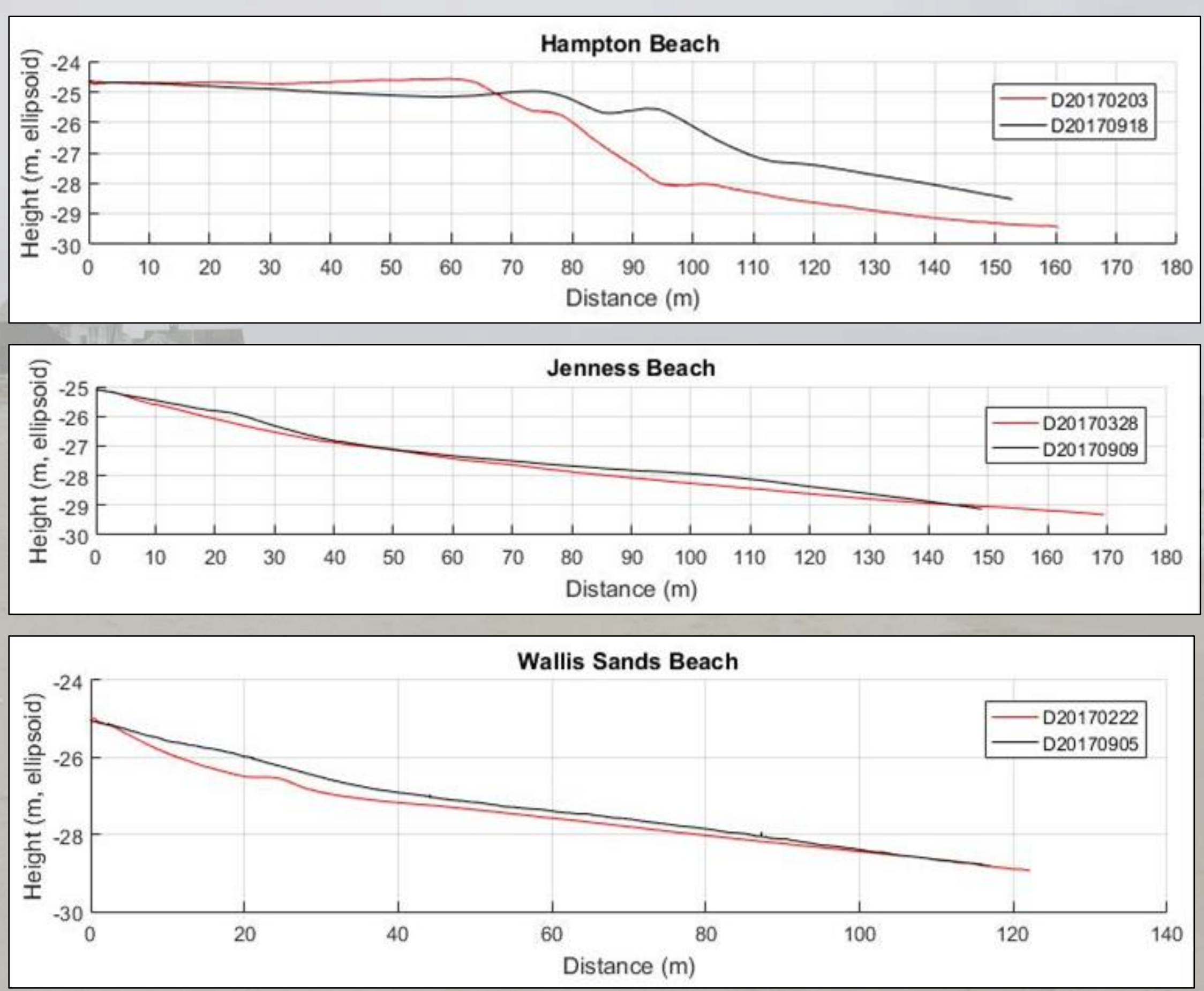


\section{Summary}

- Northern beaches are highly variable

- Alongshore and along a profile

- Bimodal with a coarser backshore

- Southern beaches are uniform

- Unimodal

- Medium to coarse sand

- Sediment sources

- On and offshore, limited longshore transport

- Eroding glacial features

- Offshore sediment bodies 


\section{Acknowledgments}

- UNH Department of Earth Science

- UNH Center for Coastal and Ocean Mapping

- BOEM - New Hampshire Cooperative Agreement

- UNH School of Marine Science

- UNH Graduate School

- New Hampshire Coastal Program 


\section{Questions?}

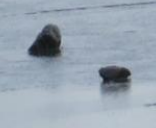

